Submitted to The Astrophysical Journal (Letters).

\title{
Hard X-ray Emission and the Ionizing Source in LINERs
}

\author{
Yuichi Terashima ${ }^{1}$, Luis C. $\mathrm{Ho}^{2}$, and Andrew F. Ptak ${ }^{3}$
}

\begin{abstract}
We report $X$-ray fluxes in the $2-10 \mathrm{keV}$ band from LINERs (low-ionization nuclear emission-line regions) and low-luminosity Seyfert galaxies obtained with the $A S C A$ satellite. Observed $X$-ray luminosities are in the range between $4 \times 10^{39}$ and $5 \times 10^{41}$ ergs $\mathrm{s}^{-1}$, which are significantly smaller than that of the "classical" low-luminosity Seyfert 1 galaxy $\mathrm{NGC} 4051$. We found that $\mathrm{X}$-ray luminosities in 2-10 keV of LINERs with broad $\mathrm{Ha}$ emission in their optical spectra (LINER 1s) are proportional to their $\mathrm{Ha}$ luminosities. This correlation strongly supports the hypothesis that the dominant ionizing source in LINER 1s is photoionization by hard photons from low-luminosity AGNs. On the other hand, the $\mathrm{X}$-ray luminosities of most LINERs without broad $\mathrm{Ha}$ emission (LINER 2s) in our sample are lower than LINER 1 s at a given $\mathrm{H} \alpha$ luminosity. The observed X-ray luminosities in these objects are insufficient to power their $\mathrm{Ha}$ luminosities, suggesting that their primary jonizing source is other than an AGN. or that an $\mathrm{AGN}$, if present, is obscured even at energies above $2 \mathrm{keV}$.
\end{abstract}

Subject headings: galaxies: active - galaxies: nuclei - galaxies: Seyfert - X-rays: galaxies

\section{Introduction}

LINERs (low-ionization nuclear emission-line regions; Heckman 1980) are fairly common in nearby galaxies. A recent optical spectroscopic survey of nearby galactic nuclei has shown that $\sim 30 \%$ of bright galaxies have LINERs (Ho, Filippenko, \& Sargent 1997a, 1997b; Ho et al. 1997c). The ionization mechanism of LINERs is still controversial (see Filippenko 1996 for a review). There are several ionization mechanisms, which can explain LINER type optical emission lines, such as (1) photoionization by low-luminosity AGNs (LLAGNs), (2) photoionization by very hot Wolf-Rayet stars or O-stars, (3) shocks, (4) cooling flows, and so on.

\footnotetext{
${ }^{1}$ ASA Goddard Space Flight Center, Code 662, Greenbelt, MD 20771

${ }^{1}$ Carnegie Observatories, 813 Santa Barbara St., Pasadena, CA 91101-1292

${ }^{1}$ Department of Physics, Carnegie Mellon University, 5000 Forbes Ave., Pittsburgh, PA 15213
} 
LLSeyfert 2s), respectively, by analogy with Seyfert 1s and Seyfert 2s. Two objects (NGC 4192 and NGC 4569) are classified as a transition object between a LINER and an H II nucleus. Although NGC 1097 was a LINER 2 historically (Phillips et al. 1984), we adopt a classification of Seyfert 1.5 based on Storchi-Bergmann, Baldwin, \& Wilson (1993) since the ASCA observation was performed after the appearance of double-peaked broad $\mathrm{Ha}$. Ha luminosities are taken from Ho et al. (1997a, c) except for several objects (see Table 1 for references). We estimated the amount of reddening by the Balmer decrement for narrow lines and corrected for reddening using the reddening curve of Cardelli et al. (1989), where we assumed the theoretical value of $\mathrm{H \alpha} / \mathrm{H} \beta=3.1$. In the case that the observed $\mathrm{Ha} / \mathrm{H} \beta$ is less than 3.1 , no correction was made. The reddening corrections for broad $\mathrm{H} \alpha$ lines were made by same amount as narrow lines.

$\mathrm{X}$-ray emission is detected from all the objects except for NGC 404. The X-ray spectra of most of the objects are represented by a two-component model consisting of an optically-thin thermal plasma with a temperature of $\sim 0.7 \mathrm{keV}$ and a hard component. Spectra of a few objects do not require a soft thermal component. The hard component is well represented by a power law with a photon index of $\sim 1.5-2.0$. Detailed results of spectral fits are given in Terashima et al. (2000b). The absorption column density for the hard component ranges from $10^{20}$ to $10^{24} \mathrm{~cm}^{-2}$. We will make use of the intrinsic luminosities of the hard component corrected for the absorption in the 2-10 keV band. If the absorption column is greater than $10^{24} \mathrm{~cm}^{-2}$ and a transmitted hard $\mathrm{X}$ ray continuum is not seen, corrections for the absorption cannot be made. In such a case, we utilize the observed luminosities corrected for only the Galactic absorption. These luminosities are summarized in Table 1. The $\mathrm{X}$-ray luminosities range from $4 \times 10^{39} \mathrm{ergs} \mathrm{s}^{-1}$ to $5 \times 10^{41} \mathrm{ergs} \mathrm{s}^{-1}$. Results of $A S C A$ observations for several objects have been already published, and references for them are also shown in Table 1.

\section{Results and Discussion}

\subsection{LINER 1s}

In order to examine whether optical emission lines in LINER 1s are photonionized by highenergy photons from an $A G N$, we search for a correlation between $X$-ray luminosities in the 2-10 $\mathrm{keV}$ band $\left(L_{\mathrm{X}}\right)$ and $\mathrm{H} \alpha$ luminosities ( $L_{\mathrm{Ha}}$ ) for objects with broad $\mathrm{H} \alpha$ in their optical spectra. It is known that a significant positive correlation between these two quantities exists for luminous AGNs (e.g., Ward et al. 1988). If photoionization by an LLAGN is the dominant jonization mechanism in LINER 1s, a $L_{\mathrm{X}}-L_{\mathrm{Ha}}$ correlation is expected. Figure 1a shows the correlation between $L_{\mathrm{X}}$ and $L_{\mathrm{Ha}}$ for LINER 1s and LLSeyfert 1s in our sample and luminous Seyfert 1s and QSOs taken from Ward et al. (1988); the $\mathrm{Ha}$ luminosities shown represent the sum of the narrow and broad components of the line. It is clear that the correlation extends to lower luminosities. The same correlation using fluxes is shown in Figure 1b. The correlation is still significant in this plot. This correlation strongly suggests that the dominant ionization source in LINER $1 \mathrm{~s}$ is photoionization by LLAGNs 
photoionization under Case B recombination and a covering fraction of unity (Osterbrock 1989). Since X-ray luminosities of most LINER $2 \mathrm{~s}$ are small $\left(L_{\mathrm{X}}<2 \times 10^{40} \mathrm{ergs} \mathrm{s}^{-1}\right)$, X-ray binaries in the host galaxy might also contribute to observed $X$-ray fluxes. Actually some objects such as NGC 3607, NGC 4111, NGC 4374, and NGC 4569 have hard band (>2 keV) images extended to several kpc (Terashima et al. 2000a, b), which implies a lower value of $L_{\mathrm{X}} / L_{\mathrm{Ha}}$ for the nuclear component. Therefore the objects with $\operatorname{low} \log L_{X} / L_{\mathrm{H} \alpha}(<1)$ are too $\mathrm{X}$-ray weak to ionize optical emission lines. even if $X$-ray variability of a factor of a few is also taken into account. If an $A G X$ is present and is the dominant ionizing source in these objects, jt should be obscured even at energies above $2 \mathrm{keV}$. Although no clear evidence for the presence of heavily obscured AGN (e.g. heavily absorbed $\mathrm{X}$-ray continuum and/or strong $\mathrm{Fe}-\mathrm{K}$ emission line) has been obtained so far, the current data cannot rule out such a possibility. Alternatively, there might be other ionization sources. The Hubble Space Telescope UV spectra of NGC 404 and NGC 4569 actually show large number of hot stars concentrated in pc-scale nuclear regions ( $\mathrm{Maoz}$ et al. 1998), and these objects could be examples of LINERs ionized by stellar sources (Terashima et al. 2000a).

\subsection{Fraction of AGNs in Bright Galaxies}

According to the optical spectroscopic survey by Ho et al. (1997a, b), Seyferts, LINER 1s and LINER $2 \mathrm{~s}$ are detected in $11 \%, 5 \%, 28 \%$ of northern $\left(\delta>0^{\circ}\right)$ bright $\left(B_{T} \leq 12.5 \mathrm{mag}\right)$ galaxies. If we assume an extreme case that all Seyferts and LINER 1 s are AGNs and all LINER $2 \mathrm{~s}$ are not AGNs. the fraction of AGN is estimated to be $16 \%$ of bright galaxies. This percentage, however, should be regarded as a lower limit because extremely weak broad $\mathrm{H} \alpha$ is difficult to detect unambiguously (Ho et al. $1997 \mathrm{c}$ ). Furthermore, some LINER 2 clearly indicate AGN-like activity. For example, NGC 4261 has prominent radio jets and kinematic evidence for a massive black hole (Ferrarese. Ford. \& Jaffe 1996). Other examples include the "Sombrero" galaxy (NGC 4594) and NGC 4736. The $L_{\mathrm{X}} / L_{\mathrm{H} \alpha}$ values for these object are similar to LINER 1s and LLSeyferts. This fact also indicates that the $L_{\mathrm{X}} / L_{\mathrm{H} \alpha}$ ratio is a good indicator of the presence of AGNs. Hard X-ray surveys conducted at high angular resolution, such as afforded by Chandra, would be crucial to refine the true AGN fraction in nearby galaxies.

The authors are grateful to all the ASCA team members. YT thanks JSPS for support. The research of LCH is partially supported by NASA grant NAG 5-3556 and by NASA grants GO-06837.01-95A and AR-07527.02-96A from the Space Telescope Science Institute (operated by AURA. Inc., under NASA contract NAS5-26555). AFP is partially supported by NASA grant NAG; $5-8093$.

\section{REFERENCES}

Allen, S. W.. Di Matteo, T.. \& Fabian, A. C. MNRAS, 2000, 311, 493 
Kriss, G. A., \& Canizares, C. R. 1982, ApJ, 261, 51

Maiolino, R. et al. 1998, A\&A, 338, 781

Makishima, K., et al. 1994, ApJ, 46, L77

Maoz, D., Koratkar, A. P., Shields, J. C., Ho, L. C., Filippenko, A. V., \& Sternberg, A. 1998, AJ, 116,55

Matsushita, K.. et al. 1994. ApJ, 436, L41

Matsumoto, H., Koyama, K., Awaki, H., Tsuru, T., Loewenstein, M., \& Matsushita, K. 1997, ApJ, 482,133

Matsumoto, Y., et al. 2000, in preparation

Mizuno, T., Ohnishi, T., Kubota, A., Makishima, K., \& Tashiro, M. 1999, PASJ, 51, 663

Mulchaey, J. S., Koratkar, A. P., Ward, M. J., Wilson, A. S., Whittle, M., Antonucci, R. R. J.. Kinney, A. L., \& Hurt, T. 1994, ApJ, 436, 586

Nicholson, K. L., Reichert, G. A., Mason, K. O., Puchnarewicz, E. W., Ho, L. C., Shields, J. C., \& Filippenko, A. V. 1998, MNRAS, 300, 893

Osterbrock, D. E. 1989, Astrophysics of Gaseous Nebulae and Active Galactic Nuclei (Mill Valley: Univ. Science Books)

Pérez-Olea, D. E. \& Colina, L. 1996, ApJ, 468. 191

Phillips, M. M., Pagel, B. E. J., Edmunds, M. G., \& Díaz, A. 1984, MNRAS, 210, 701

Ptak, A., Serlemitsos, P. J., Yaqoob, T., \& Mushotzky, R. 1999, ApJS, 120, 179

Ptak, A., Yaqoob, T., Serlemitsos, P. J., Kunieda, H., \& Terashima, Y. 1996, ApJ, 459, 542

Roberts, T. P., Warwick, R. S.. \& Ohashi, T. 1999, MNRAS, 304, 52

Sambruna. R. M., Eracleous, M., \& Mushotzky, R. F. 1999, ApJ, 526, 60

Shields. J. C. et al. 1999. ApJL, submitted

Storchi-Bergmann, T.. Baldwin, J. A., \& Wilson, A. S. 1993, ApJ, 410, L11

Storchi-Bergmann, T., Eracleous, M., Livio, M., Wilson, A. S., Filippenko, A. V., \& Halpern. J. P. 1995. ApJ, 443.617

Storchi-Bergmann, T. \&: Pastriza, M.G. 1989. ApJ, 347, 195

Taniguchi, Y., Ohyama. Y., Yamada, T., Mouri, H., \& Yoshida, M. 1996, ApJ, 467, 215 
Table 1. X-ray and $\mathrm{H} \alpha$ luminosities for observed galaxies

\begin{tabular}{|c|c|c|c|c|c|c|c|c|}
\hline Name & $\begin{array}{l}\text { Distance } \\
{[\mathrm{Mpc}]}\end{array}$ & Class & $\begin{array}{c}\log L(\mathrm{Ha}) \\
\text { (Broad) } \\
{\left[\text { ergs s}^{-1}\right]}\end{array}$ & $\begin{array}{l}\log L(\mathrm{H} \alpha) \\
(\text { Narrow) } \\
{\left[\text { ergs s}^{-1}\right]}\end{array}$ & $\begin{array}{c}\log L_{\mathrm{X}} \\
(2-10 \mathrm{keV}) \\
{\left[\text { ergs s}{ }^{-1}\right]}\end{array}$ & $L_{\mathrm{X}} / L_{\mathrm{H} \alpha}$ & $\begin{array}{c}\text { refere } \\
X\end{array}$ & $\begin{array}{l}\mathrm{Ha} \\
\mathrm{Ha}\end{array}$ \\
\hline NGC 315 & 65.8 & L1.9 & 39.92 & 39.61 & 41.70 & 2.09 & 1 & \\
\hline NGC 1052 & 17.8 & L1.9 & 39.54 & 39.45 & 41.58 & 2.13 & 2,3 & 1,2 \\
\hline NGC 3998 & 21.6 & L1.9 & 40.59 & 40.43 & 41.67 & 1.24 & 4 & \\
\hline NGC 4203 & 9.7 & L1.9 & 38.59 & 38.34 & 40.40 & 2.06 & 5 & \\
\hline NGC 4438 & 16.8 & L1.9 & 39.54 & 40.04 & 39.96 & -0.08 & & \\
\hline NGC 4450 & 16.8 & $\mathrm{~L} 1.9$ & 38.48 & 38.51 & 40.34 & 1.83 & & 1.2 \\
\hline NGC 4579 & 16.8 & S1.9/L1.9 & 39.49 & 39.53 & 41.18 & 1.65 & 4,6 & \\
\hline NGC 4636 & 17.0 & L1.9 & 38.37 & 38.27 & 40.22 & 1.95 & $7-11$ & \\
\hline NGC 5005 & 21.3 & L1.9 & 40.05 & 39.48 & 40.59 & 1.11 & & \\
\hline NGC 404 & 2.4 & L2 & $\ldots$ & 37.82 & $<37.66$ & $<-0.16$ & 12 & \\
\hline NGC 3507 & 19.8 & $\mathrm{~L} 2$ & $\ldots$ & 39.60 & 39.92 & 0.32 & & \\
\hline NGC 3607 & 19.9 & $\mathrm{~L} 2$ & $\ldots$ & 39.53 & 40.16 & 0.63 & & \\
\hline NGC 4111 & 17.0 & $\mathrm{~L} 2$ & $\ldots$ & 39.85 & 39.94 & 0.09 & 12 & \\
\hline NGC 4192 & 16.8 & $\mathrm{~T} 2$ & $\ldots$ & 40.49 & 39.58 & -0.91 & 12 & \\
\hline NGC 4261 & 35.1 & $\mathrm{~L} 2$ & $\ldots$ & 39.82 & 41.18 & 1.36 & 1. 13 & \\
\hline $\mathrm{NGC} 4374$ & 16.8 & $\mathrm{~L} 2$ & $\ldots$ & 39.35 & 40.33 & 0.98 & $8-10$ & \\
\hline NGC 4457 & 17.4 & $\mathrm{~L} 2$ & $\ldots$ & 39.79 & 39.98 & 0.20 & 12 & \\
\hline NGC 4569 & 16.8 & $\mathrm{~T} 2$ & $\ldots$ & 40.66 & 40.08 & -0.58 & 12 & 3 \\
\hline NGC 4.594 & 20.0 & $\mathrm{~L} 2$ & $\ldots$ & 39.82 & 41.00 & 1.18 & 4.14 & \\
\hline NGC 4736 & 4.3 & $\mathrm{~L} 2$ & $\ldots$ & 38.12 & 39.64 & 1.52 & 15 & 4 \\
\hline NGC 7217 & 16.0 & $\mathrm{~L} 2$ & $\ldots$ & 39.78 & 39.86 & 0.08 & & \\
\hline NGC $109 i$ & 14.5 & $\mathrm{~S} 1.5$ & 40.55 & 39.53 & 40.63 & 1.10 & 16 & 5 \\
\hline NGC 1365 & 16.9 & S1.8 & 42.02 & 41.08 & 40.50 & -0.58 & 17 & 6 \\
\hline NGC 2639 & 42.6 & S1.9 & 39.79 & 40.48 & 41.66 & 1.18 & 18 & 1.2 \\
\hline NGC 3031 & 1.4 & $\mathrm{~S} 1.5$ & 38.41 & 38.44 & 39.60 & 1.16 & 19 & \\
\hline NGC. 4258 & 6.8 & S1.9 & 38.90 & 38.60 & 40.83 & 2.23 & 4,20 & \\
\hline NGC 4565 & $9 . \overline{7}$ & S1.9 & 38.38 & 38.46 & 39.77 & 1.31 & 21 & \\
\hline NGC 4639 & 16.8 & $\mathrm{~S} 1.0$ & 39.75 & 38.39 & 40.54 & 2.15 & 22 & \\
\hline NGC 5033 & 18.7 & $\mathrm{~S} 1.5$ & 40.32 & 39.70 & 41.37 & 1.67 & 23 & \\
\hline NGC 1386 & 16.9 & $\mathrm{~S} 2$ & $\ldots$ & 40.94 & 40.34 & -0.60 & 17 & $\bar{T}$ \\
\hline $\mathrm{NGC} 2273$ & 28.4 & $S 2$ & $\ldots$ & 41.00 & 41.93 & 0.93 & & \\
\hline
\end{tabular}




\section{Figure Captions}

Fig. 1.- (a) Correlation between X-ray and $\mathrm{H} \alpha$ luminosities for LINER 1s, low-luminosity Seyfert 1s, and luminous type 1 AGNs taken from Ward et al. (1998)., (b) Correlation between X-ray and $\mathrm{H} \alpha$ fluxes for the same sample as $(a)$. The triangle and the cross correspond to NGC 1365 and $\mathrm{NGC} 4438$, respectively.

Fig. 2.- $\log L_{\mathrm{X}} / L_{\mathrm{H \alpha}}$ for low-luminosity Seyfert 1s, low-luminosity Seyfert 2s, LINER 1s, and LINER 2s. The symbol "<" denotes an upper limit. 


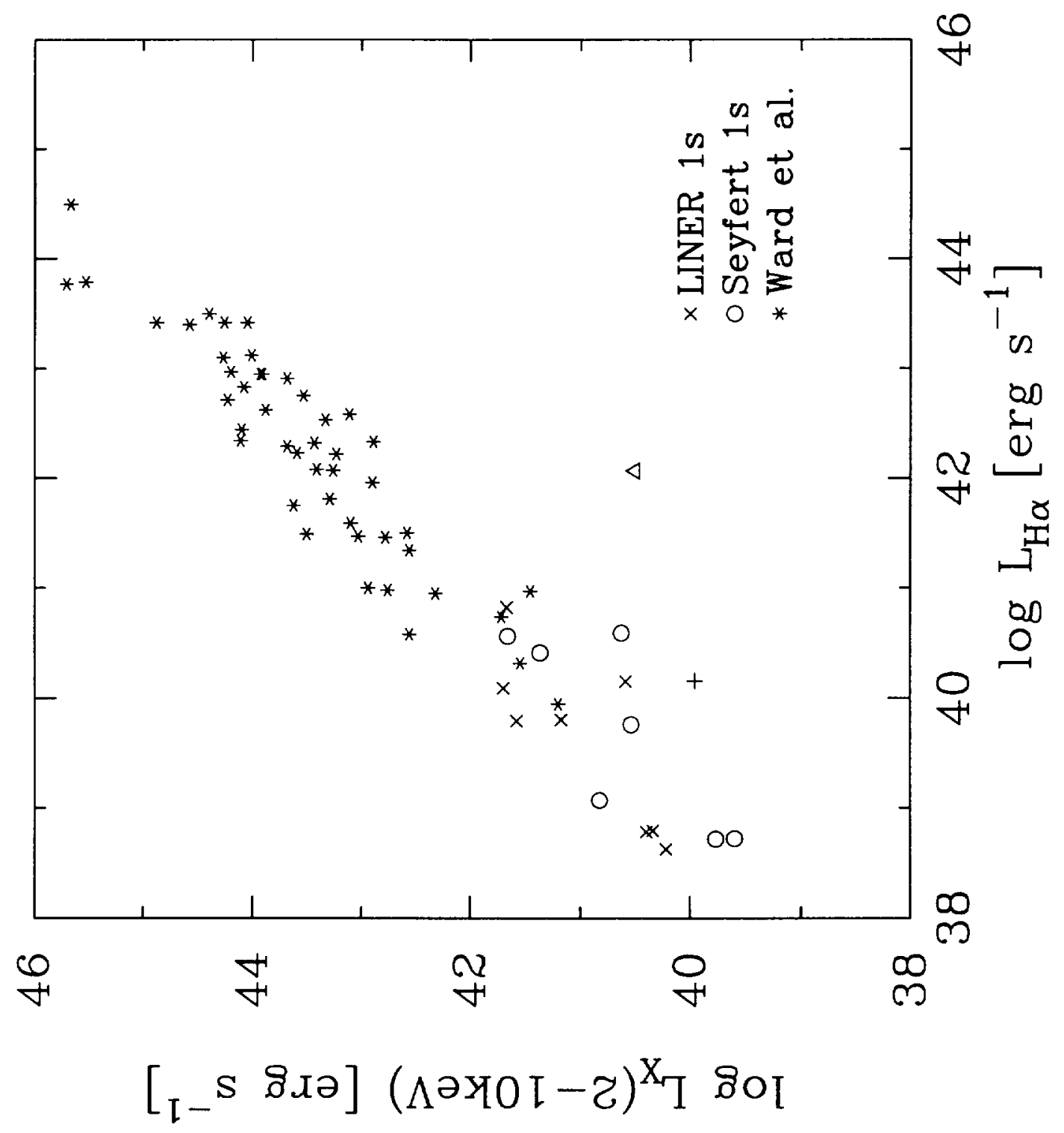




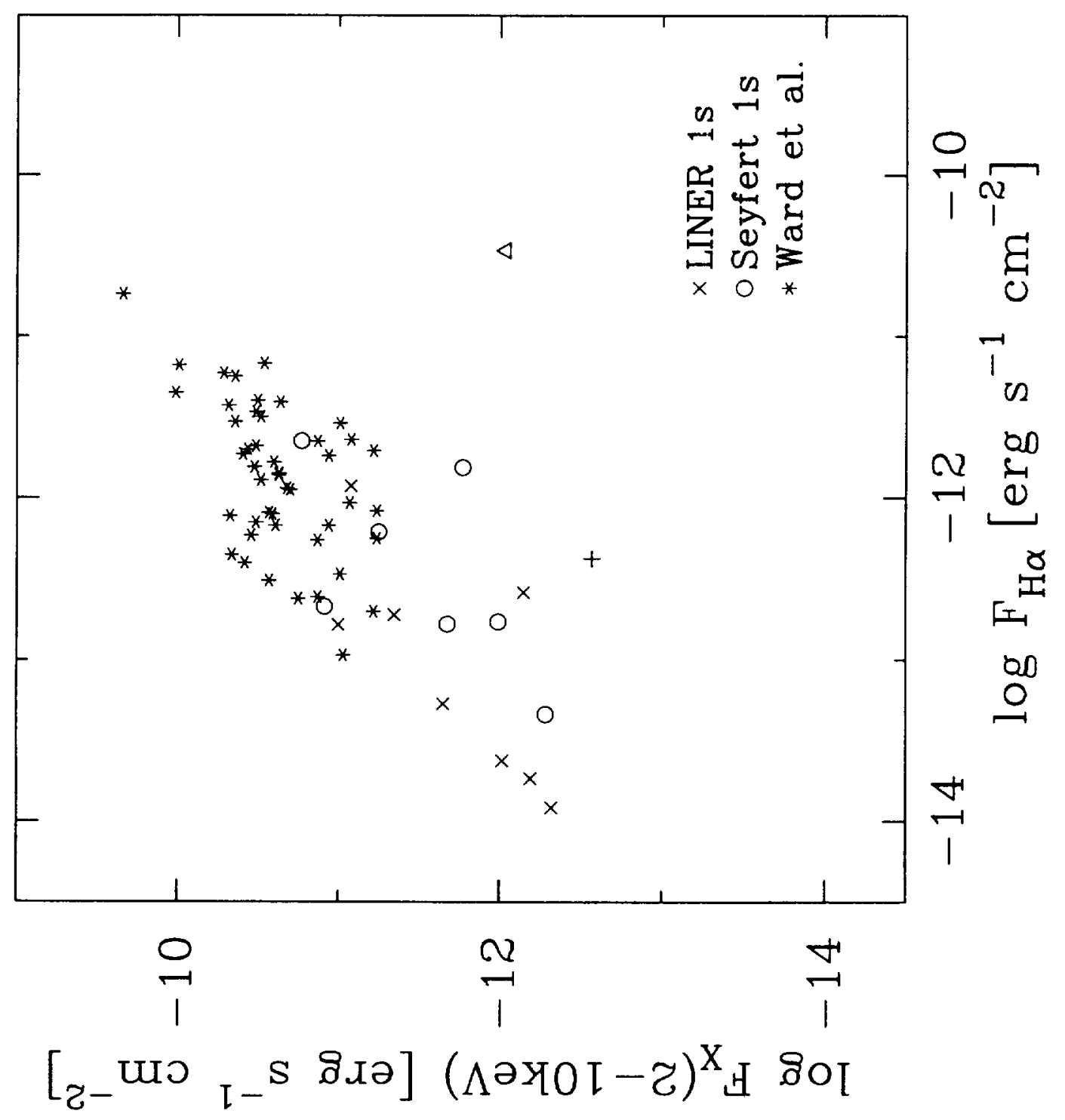




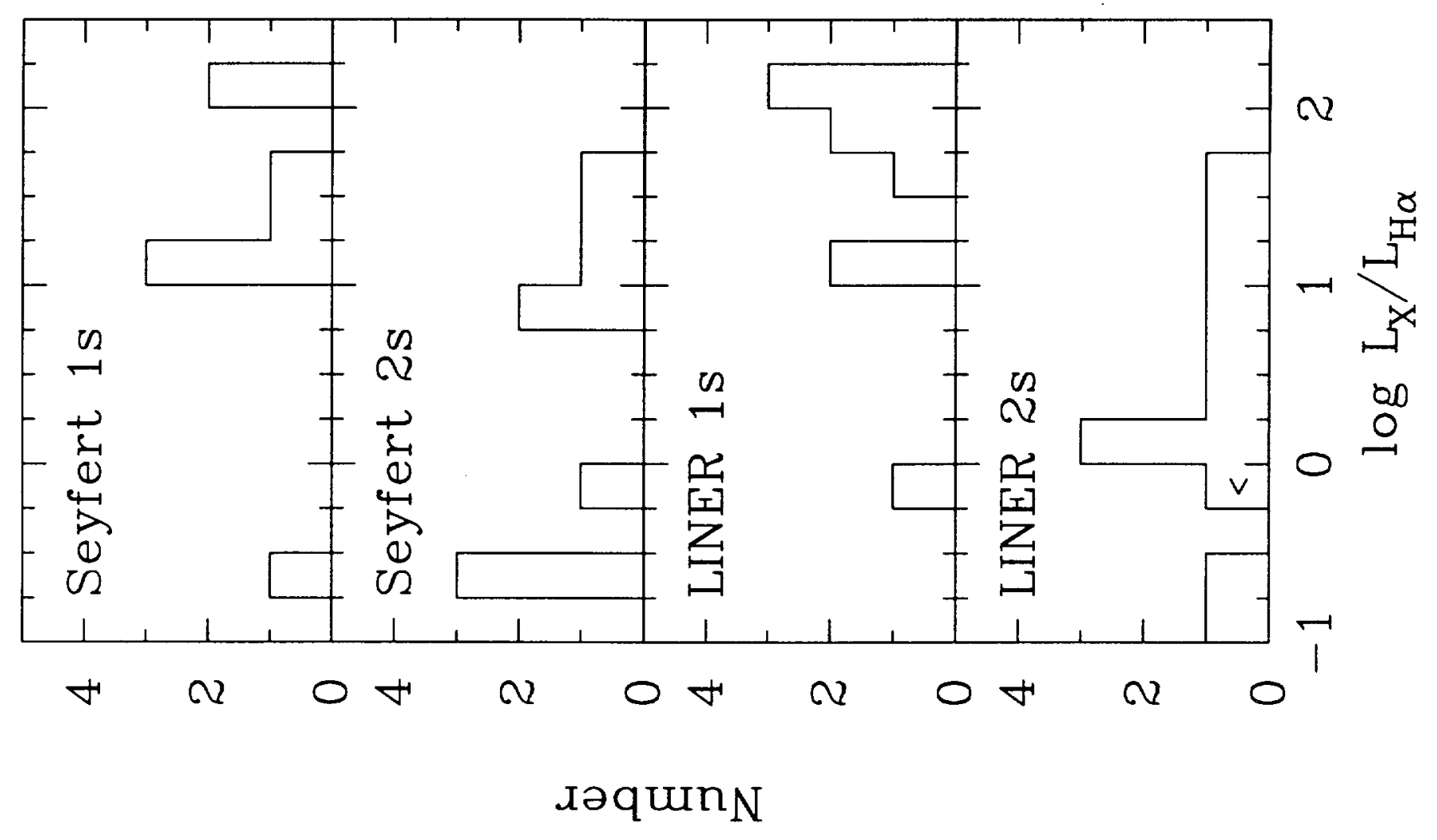

